\section{Commentary: Swift reaction to malperfusion saves lives}

\author{
Tohru Asai, MD, PhD
}

Acute type A aortic dissection, or AADA, is a lifethreatening condition, with a $1 \%$ to $2 \%$ increase of mortality per hour soon after symptom onset. Early surgical central aortic repair has been established as a treatment of choice, with low hospital mortality reported. ${ }^{1}$ However, preoperative organ malperfusion syndrome (MPS) has remained a serious complication that influences mortality and morbidity. Optimal management strategies for each organ malperfusion have been proposed but have not been well established.

In this issue of the Journal, Nakai and colleagues ${ }^{2}$ present data reviewing their experience with surgical repair for AADA complicated with preoperative MPS. Among 331 surgically treated patients with AADA, $84(25 \%)$ had preoperative malperfusion. Patients reached the operating room within 5 hours from symptom onset in $69 \%$ of cases (the immediate group), or beyond 5 hours in $31 \%$ (the later group). While the authors found that preoperative coronary malperfusion was the only predictor of early mortality, the early mortality rates were not significantly different between the 2 groups. In contrast, cumulative 5-year survival rate in the immediate group was $76.7 \%$, versus those in the later group, at $45.4 \%$, with coronary malperfusion and shock on arrival being associated with lower rates of long-term survival. The difference in long-term survival was not evident among patients without preoperative MPS. Nakai and colleagues conclude that performing a surgical repair within 5 hours from onset of symptom improves long-term outcomes in patients with AADA and MPS.

Malperfusion complicating AADA affects many different organs in different ways. The tolerable ischemic

From the Department of Cardiovascular Surgery, Juntendo University, Tokyo, Japan. Disclosures: The author reported no conflicts of interest.

The Journal policy requires editors and reviewers to disclose conflicts of interest and to decline handling or reviewing manuscripts for which they may have a conflict of interest. The editors and reviewers of this article have no conflicts of interest.

Received for publication April 7, 2021; revisions received April 7, 2021; accepted for publication April 7, 2021; available ahead of print April 20, 2021.

Address for reprints: Tohru Asai, MD, PhD, Faculty of Medicine, Department of Cardiovascular Surgery, Juntendo University, 2-1-1, Hongo, Bunkyo-ku, Tokyo 113-8421, Japan (E-mail: t.asai.jj@juntendo.ac.jp).

J Thorac Cardiovasc Surg 2023;165:992-3

$0022-5223 / \$ 36.00$

Copyright (c) 2021 by The American Association for Thoracic Surgery

https://doi.org/10.1016/j.jtcvs.2021.04.017

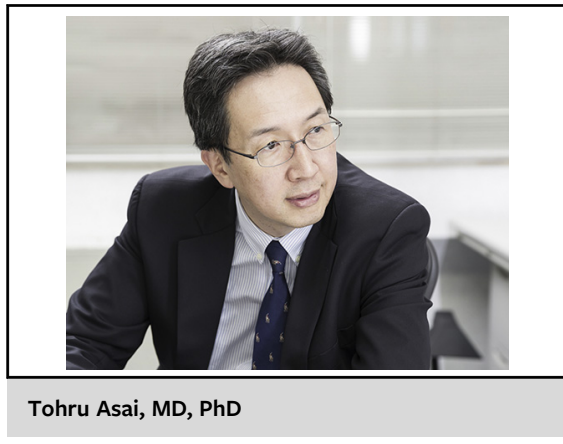

CENTRAL MESSAGE

The sooner the central repair is performed, the better the longterm survival in patients with acute type $\mathrm{A}$ aortic dissection with malperfusion, particularly coronary malperfusion.

times of organs vary. That time may be very short in coronary malperfusion and even shorter with cerebral malperfusion, risking stroke and coma. These can be fatal if not treated immediately. Aortic repair may alleviate malperfusion to distal aortic branches, possibly removing any need for further revascularization. Impacts of organ damage clearly vary in MPS. Therapeutic strategies for AADA complicated by MPS are still matters of controversy, particularly whether early revascularization should precede central aortic repair. Nakai's group has adopted the protocol to perform immediate central aortic repair in most cases of AADA with MPS, with the possible exception of angioplasty first in cases of coronary malperfusion.

The sooner the central repair for AADA is performed, the better the probable long-term survival, particularly in patients with MPS. But how can we further improve the clinical outcomes? A key point may be to establish more sophisticated organ-specific management, particularly in cases with coronary, brain, and mesenteric malperfusion, whether central aortic repair ${ }^{3}$ or early reperfusion ${ }^{4}$ should take priority.

Clearly, every effort should be made to avoid diagnosis delay in AADA with MPS. I am grateful to Nakai and colleagues for attracting the attention of our readers to the importance of timing from the onset of symptoms for managing AADA compromised by malperfusion. 


\section{References}

1. Evangelista A, Isselbacher EM, Bossone E, Gleason TG, Eusanio MD, Sechtem U, et al. Insights from the international registry of acute aortic dissection: a 20-year experience of collaborative clinical research. Circulation. 2018;137:1846-60.

2. Nakai C, Izumi S, Haraguchi T, Henmi S, Nakayama S, Mikami T, et al. Impact of time from symptom onset to operation on outcome of repair of acute type-A aortic dissection with malperfusion. J Thorac Cardiovasc Surg. 2023;165:984-91.e1.
3. Chiu P, Tsou S, Goldstone AB, Louie M, Woo YJ, Fischbein MP. Im mediate operation for acute type A aortic dissection complicated by visceral or peripheral malperfusion. J Thorac Cardiovasc Surg. 2018; 156:18-24.

4. Uchida K, Karube N, Kasama K, Minami T, Yasuda S, Goda M, et al. Early reperfusion strategy improves the outcomes of surgery for type A acute aortic dissection with malperfusion. J Thorac Cardiovasc Surg. 2018;156:483-9.
See Article page 984 .

\section{Commentary: Timely repair of acute aortic dissection: Every minute counts}

\author{
Aly Ghoneim, $\mathrm{MD}, \mathrm{PhD},{ }^{\mathrm{a}}$ \\ Maral Ouzounian, MD, $\mathrm{PhD}{ }^{\mathrm{b}}$ \\ Mark D. Peterson, MD, PhD, ${ }^{b}$ \\ Ismail El-Hamamsy, $\mathrm{MD}, \mathrm{PhD}$, \\ Francois Dagenais, MD, ${ }^{\mathrm{d}}$ and \\ Michael W. A. Chu, MD, MEd, ${ }^{\text {a }}$ on behalf of the \\ Canadian Thoracic Aortic Collaborative (CTAC)
}

Acute type A aortic dissection (ATAAD) with malperfusion remains a lethal emergency with high morbidity and mortality rates. Data from the International Registry of Acute Aortic-Dissection revealed an incremental risk of death $(1 \%-2 \%)$ for every hour without immediate repair after symptom onset. ${ }^{1}$ Rapid assessment, immediate medical optimization, and early surgery are lifesaving and likely to improve early and late clinical outcomes., ${ }^{2,3}$

In this issue of the Journal, Nakai and colleagues ${ }^{4}$ investigated the importance of timing from symptom onset to surgical repair in patients with ATAAD complicated by malperfusion syndrome. They found that surgery within

\footnotetext{
From the ${ }^{\mathrm{a}}$ Western University, London, Ontario, Canada; ${ }^{\mathrm{b}}$ University of Toronto, Toronto, Ontario, Canada; ${ }^{\mathrm{c}}$ Mount Sinai Hospital and Icahn School of Medicine at Mount Sinai, New York, NY; and ${ }^{\mathrm{d}}$ Institut universitaire de cardiologie et de pneumologie de Québec, Université Laval, Quebec City, Quebec, Canada.

Disclosures: The authors reported no conflicts of interest.

The Journal policy requires editors and reviewers to disclose conflicts of interest and to decline handling or reviewing manuscripts for which they may have a conflict of interest. The editors and reviewers of this article have no conflicts of interest.

Received for publication April 11, 2021; revisions received April 11, 2021; accepted for publication April 12, 2021; available ahead of print April 17, 2021.

Address for reprints: Michael W.A. Chu, MD, MEd, B6-106 University Hospital, London Health Sciences Centre, 339 Windermere Rd, London, Ontario, Canada, N6A 5A5 (E-mail: Michael.Chu@1hsc.on.ca).

J Thorac Cardiovasc Surg 2023;165:993-4

$0022-5223 / \$ 36.00$

Copyright (c) 2021 by The American Association for Thoracic Surgery

https://doi.org/10.1016/j.jtcvs.2021.04.026
}

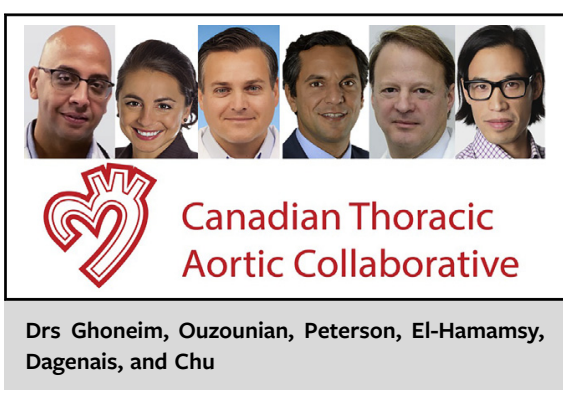

CENTRAL MESSAGE

Acute aortic dissection is a lifethreatening condition in which survival is critically linked to timeliness of definitive surgical repair.

5 hours of symptom onset was a predictor of long-term survival. Although their analysis was limited by smaller patient numbers, the authors contribute additional important data that associate earlier surgery with improved outcomes. The authors should be congratulated for identifying the exact timing of symptom onset, a parameter that previous studies often estimate, allowing the authors to carefully link the timing of the dissection to definitive therapy. It was impressive that they were able to get their early-surgery patients $(69 \%)$ into the operating room within a mean of 3.5 hours from symptom onset, which may limit the generalizability of their findings. Although early mortality and complication rates were not different, the intermediate and late survival was significantly better in the early-surgery group. Unfortunately, no information about late aortic-remodeling or aortic outcomes was presented to explain how this survival difference was related to the initial dissection repair. More data are required to understand better the authors' approach to coronary 